\title{
Contingent commodities and implementation
}

\author{
Subir Chattopadhyay ${ }^{\mathrm{a}}$, Luis Corchon ${ }^{\mathrm{b}, *}$, Jorg Naeve $^{\mathrm{c}}$ \\ ${ }^{\mathrm{a}}$ Universidad de Alicante, Alicante, Spain \\ ${ }^{\mathrm{b}}$ Departamento Fundamentos del Analisis Economico, Universidad Carlos III de Madrid, E-28093 Getafe, Spain \\ ${ }^{\mathrm{c}}$ University of Bielefeld, Bielefeld, Germany
}

Received 24 September 1999; accepted 7 January 2000

\begin{abstract}
In this note we study the relevance of using contingent commodity allocations when states are not directly contractible. In such a setting, a contingent commodity allocation takes the form of a social choice function, and the question is whether this function is implementable. Using only very mild assumptions on the rule for selecting contingent commodity allocations, we derive a strong negative result which proves to be robust with respect to different solution concepts employed for implementation. These findings have interesting implications for the interpretation of Arrow-Debreu economies. (C) 2000 Elsevier Science S.A. All rights reserved.
\end{abstract}

Keywords: Contingent commodities, Implementation, Single-crossing

JEL classification: D50

\section{Introduction}

The Arrow-Debreu model of general equilibrium with state contingent commodities is often considered to be a benchmark for the realization of efficient resource allocation; in recent years its usage has become pervasive, particularly in macroeconomics. The cautionary tales that are told regarding the suitability of the model are usually framed in terms of imperfections like nonconvexities, thin markets, and asymmetries of information among agents. But it is accepted that if the economic situation to be modeled is immune to these imperfections, and the states of nature are 'macroeconomic,' then the Arrow-Debreu model is an acceptable approximation to reality. Our concern here is a precise formulation of the idea that a state is macroeconomic and we make the point that for the model to 'work,' the states have to be contractible. In this note we explore the consequences of assuming that states are not contractible. In this case, we need a mechanism that

*Corresponding author. Tel.: +34-91-624-9617; fax: +34-91-624-9875.

E-mail address: lcorchon@eco.uc3m.es (L. Corchon) 
generates, or implements, the outcomes of the Arrow-Debreu model as the equilibria of a game without introducing any new outcomes. As is well known, the (interior) Walrasian correspondence can be implemented in Nash equilibrium so it would appear that, by a standard extension of the commodity space using contingent commodities, so can Arrow-Debreu equilibrium allocations. We show that such is not the case. We follow up on an early example due to Palfrey and Srivastava (1989, p. 129) and show that the basic intuition given by them holds much more generally. Our point is very simple, almost trivial: If preferences are state independent, any equilibrium concept yields identical ex post incentives at every state so any implementable contingent commodity allocation must be constant in the state. Given that there are economies for which no interior ex ante Pareto efficient rule satisfies this requirement, it follows that any such rule is not implementable. We emphasize that our result goes through to all the standard game theoretical equilibrium concepts (so the consideration of stage games and subgame perfection does not help), and to any ex ante Pareto efficient rule that satisfies an interiority assumption.

One can ask what we learn from such a result. One always pauses and gives some thought to the appropriateness of using the Arrow-Debreu model in a particular situation; our result allows one to have a better grasp on the said appropriateness since it says that the Arrow-Debreu model can be used provided that states are verifiable (earthquakes and other natural calamities, etc.). In other words, the relevant asymmetry of information is between agents and courts and not just between agents as emphasized in the earlier literature (see, for example, Radner, 1968).

\section{Model and the results}

We consider the standard model of an Arrow-Debreu economy with uncertainty. We have a finite set $\mathscr{I}=\{1, \ldots, I\}$ of agents and a finite set $\mathscr{L}=\{1, \ldots, L\}$ of physically different goods. The economy exists for two periods. At date 0 there is uncertainty about which of the possible states of the world from the finite set $\mathscr{S}=\{1, \ldots, S\}$ will be realized at date 1 , while we assume that at that date realized state of the world will become common knowledge among the agents. The commodity space is $\mathscr{R}^{L \cdot S}$, a typical element of which is a contingent commodity vector $x=$ $\left(x_{1,1}, \ldots, x_{L, 1}, \ldots, x_{1, S}, \ldots, x_{L, S}\right)$, where the component $x_{l, s}$ is to be interpreted as $x_{l, s}$ units of good $l$ if state $s$ occurs at date 1 . We shall use superscripts to refer to agents in the economy. Hence, an allocation in this economy is a list, $\left(x^{1}, \cdots, x^{I}\right)$, where $x^{i} \in \mathscr{R}^{L \cdot S}$ for all $i \in \mathscr{I}$. Sometimes it will be convenient to have the following shorthand notation available: Let $x \in \mathscr{R}^{L \cdot S}$ and $s \in \mathscr{S}$, then we write $x_{s}=\left(x_{1, s}, \ldots, x_{L, s}\right), x_{s} \in \mathscr{R}^{L}$; analogously, we write $x_{l} \in \mathscr{R}^{S}$.

Each agent $i$ is characterized by a consumption set $X^{i}=\mathscr{R}_{+}^{L \cdot S}$, an initial endowment $\omega^{i} \in \mathscr{R}_{+}^{L \cdot S}$, and preferences representable by a utility function $u^{i}(x)=\Sigma_{s \in \mathscr{S}} q^{i}(s) v^{i}\left(x_{s}, s\right)$, where $q^{i}$ is the subjective probability distribution over the states. We assume that $v^{i}(\cdot, s)$ is strictly monotonic and once continuously differentiable $\left(C^{1}\right)$.

We assume that the aggregate endowment does not vary with the state of the world. Thus, the set of feasible allocations in, say, states $s$ and $t$ are identical and we denote it by $\mathscr{A}$. A contingent commodity allocation can be now written as a Social Choice Function $\mathscr{X}: \mathscr{S} \rightarrow \mathscr{A}, \mathscr{X}(s)=\left(x_{s}^{1}, \ldots, x_{s}^{I}\right)$.

The usual way of interpreting a contingent commodity allocation $\mathscr{X}$ is as a contract signed at date 0 , i.e. when agents know their preferences but the state of the world to prevail at date 1 has not been 
determined yet. At date 1, every agent receives the quantity specified by such a contract. So, in this interpretation, states are assumed to be 'contractible'. Since we wish to explore the consequences of dispensing with that assumption, the question that interests us is: Given a contingent commodity allocation, is it possible to design a mechanism in such a way that for every state the equilibrium messages of the agents lead to the outcome prescribed by the contingent commodity allocation for that state?

Notice that, contrary to the usual implementation problem, we will assume that ex ante preferences are fixed and given; what changes, the underlying 'economy', is the Arrow-Debreu state of the world which determines ex post preferences.

A mechanism takes the form $g=(M, f)$, where $M=\prod_{i \in \mathscr{I}} M^{i}$ is the message space and $f$ : $M \rightarrow \mathscr{R}^{L \cdot I}$ the outcome function. The messages are meant to convey information concerning the realized state of the world, the outcome function then determining an allocation for that state. Given agents' preferences, and a state $s$, the mechanism $g$ induces a non-cooperative game $\Gamma_{s}$ in which $M^{i}$ is player $i$ 's strategy set and her payoff function is given by $v^{i}(\cdot, s) \circ f$.

Definition 2.1. Let $E(s)$ denote the set of equilibria of $\Gamma_{s}$ for a given equilibrium concept. ${ }^{1}$ We say that a mechanism $g$ implements the social choice function $\mathscr{X}$ in that equilibrium concept if, for all states $s \in \mathscr{S}$, we have

$$
f(\mathrm{E}(s))=\mathscr{X}(s)
$$

We first notice the following:

Remark 1. Assume that agents have state-independent utility functions, i.e.

$$
u^{i}(x)=\sum_{s \in \mathscr{S}} q^{i}(s) v^{i}\left(x_{s}^{i}\right)
$$

Let $\Phi: \mathscr{S} \rightarrow \mathscr{R}^{L \cdot I}$ be a social choice correspondence. If $\Phi$ is implementable (in any equilibrium concept) it is constant, that is, $\Phi(s)=\Phi(t) \forall s, t \in \mathscr{S}$.

Proof. Let $g=(M, f)$ be a mechanism implementing $\Phi$ so that we have

$$
f(\mathrm{E}(s))=\Phi(s) \quad \forall s \in \mathscr{S}
$$

Given the assumption on agents' preferences, for any two states $s$ and $t$, the non-cooperative games $\Gamma_{s}$ and $\Gamma_{t}$ induced by $g$ coincide because the payoff function for, say, agent $i$ is $v^{i} \circ f$. Therefore, the sets of equilibria of these games are identical,

$$
E(s)=E(t) \quad \forall s, t \in \mathscr{S}
$$

and, thus, from Eq. (1) above,

$$
\Phi(s)=\Phi(t) \quad \forall s, t \in \mathscr{S}
$$

\footnotetext{
${ }^{1}$ For good general discussions of implementation, see Maskin (1985) or Moore (1992). See also Matsushima (1988) and Abreu and Sen (1991) for virtual implementation.
} 
Next we show that there are economies with state-independent spot market utility functions for which no interior ex ante Pareto optimal contingent commodity allocation is constant; hence, there exists a conflict between implementability and ex ante Pareto efficiency (for interior allocations).

Proposition. There exist economies $\mathscr{E}=\left(\left(X^{i}, u^{i}, \omega^{i}\right)_{i \in \mathfrak{A}}\right)$ such that no ex ante Pareto optimal, interior contingent commodity allocation is implementable.

Proof. We will construct such an economy. For $i \in \mathscr{I}$, let $X^{i}=\mathscr{R}_{+}^{L \cdot S}$ and let the utility function $u: \mathscr{R}_{+}^{L \cdot S} \rightarrow \mathscr{R}$ be defined by

$$
u^{i}(x)=\sum_{s \in \mathscr{S}} q^{i}(s) v\left(x_{s}^{i}\right)
$$

where $v: \mathscr{R}_{+}^{L} \rightarrow \mathscr{R}$ is monotone, strictly concave, state independent and differentiable. Consider two states of the world $s, s^{\prime} \in S$ and let $q^{i}(s) q^{j}\left(s^{\prime}\right) \neq q^{i}\left(s^{\prime}\right) q^{j}(s)$ for some $i, j \in \mathscr{I}$. For all other states the probabilities can be chosen arbitrarily.

Let $\left(\bar{x}^{1}, \ldots, \bar{x}^{I}\right) \in \mathscr{R}^{L \cdot S \cdot I}$ be an interior Pareto optimal allocation. Then $\left(\bar{x}^{1}, \ldots, \bar{x}^{I}\right)$ solves the following maximization problem for some $\left(\alpha^{1}, \ldots, \alpha^{T}\right), \alpha \gg 0$ and $\Sigma_{i \in \mathscr{I}} \alpha^{i}=1$.

$$
\begin{aligned}
& \max _{\left(\bar{x}^{1}, \quad \bar{x}^{I}\right) \in \mathscr{R}} \sum_{+} \sum_{+} \alpha^{i} \sum_{s \in \mathscr{S}} q^{i}(s) v\left(\bar{x}_{s}^{i}\right) \\
& \text { s.t. } \sum_{i \in \mathscr{I}}\left(\omega^{i}-\bar{x}^{i}\right) \geq 0
\end{aligned}
$$

The first-order conditions for this problem are

$$
\frac{\frac{\partial v}{\partial x_{l}}\left(\bar{x}_{s}^{i}\right)}{\frac{\partial v}{\partial x_{l}}\left(\bar{x}_{s}^{j}\right)}=\frac{\alpha^{j}}{\alpha^{i}} \frac{q^{i}(s)}{q^{j}(s)}
$$

Now consider the two states $s, s^{\prime} \in \mathscr{S}$ identified above. If the allocation $\left(\bar{x}^{1}, \ldots, \bar{x}^{I}\right)$ assigned the same spot market allocation in both states, we would have that for all $i, j \in \mathscr{I}, i \neq j$, and for all $l \in \mathscr{L}$,

$$
\frac{\frac{\partial v}{\partial x_{l}}\left(\bar{x}_{s}^{i}\right)}{\frac{\partial v}{\partial x_{l}}\left(\bar{x}_{s}^{j}\right)}=\frac{\frac{\partial v}{\partial x_{l}}\left(\bar{x}_{s^{\prime}}{ }^{\prime}\right)}{\frac{\partial v}{\partial x_{l}}\left(\bar{x}_{s^{\prime}}{ }^{\prime}\right)}
$$

while by assumption

$$
\frac{q^{i}(s)}{q^{j}(s)} \neq \frac{q^{i}\left(s^{\prime}\right)}{q^{j}\left(s^{\prime}\right)}
$$

This would lead to a contradiction. 
Therefore, it must be the case that $\left(\bar{x}_{s}{ }^{1}, \ldots, \bar{x}_{s}^{I}\right) \neq\left(\bar{x}_{s}{ }^{\prime}, \ldots, \bar{x}_{s}{ }^{\prime}\right)$. But then it follows from Remark 1 that $\left(\bar{x}^{1}, \ldots, \bar{x}^{I}\right)$ is not implementable.

Several comments are in order: Firstly, the assumption that utility functions are state independent can be generalized to utility function being state monotonic, i.e. for any pair of states $s$ and $t$, there is a strictly increasing function $F_{s t}$ such that $v(\cdot, s)=F_{s t}(v(\cdot, t))$. Secondly, if we look for positive results, we have to allow for state-dependent utility functions. The condition of Maskin Monotonicity (Maskin, 1977) is necessary and, under our assumptions, sufficient for Nash implementability with three or more agents. As Maskin himself notes the well-known strict Spence-Mirrlees single-crossing property is a sufficient condition for Maskin monotonicity to be satisfied. The strict single-crossing property admits an economic interpretation. As an example, let us assume two goods - umbrellas and sun-lotion - and let there be two states of the world, rain and sunshine. If agents' marginal rates of substitution between umbrellas and sun-lotion change unambiguously with the weather, everybody being willing to exchange more sun-lotion for an umbrella in case it rains than on a hot and sunny summer day, strict single-crossing is satisfied. Finally, notice that our result deals with situations in which efficient rules can not be implemented because of adverse selection problems (and not because of moral hazard problems as in Bernheim and Whinston, 1998).

\section{Concluding remarks}

How should the results we have obtained be interpreted? In the case in which utilities are state independent and additively separable across states (a case usually assumed in the literature), they tell us that one needs to assume that one can write contracts on the states of the world directly. In other words, the use of contingent commodities presupposes the existence of some institution which is able of verifying the state of the world and to enforce transactions agreed on ex ante while the lack of such an institution imposes common priors when preferences are state independent. However, if utilities are state dependent and satisfy the strict single crossing property, Nash implementation of Arrow-Debreu equilibrium becomes possible.

Given the observation that, in general, use of the Arrow-Debreu model assumes the existence of an institution able to enforce contingent commodity allocations, it may be worthwhile to reconsider the model of Radner (1968) in which agents are endowed with information structures, i.e. partitions of the set of possible states of the world, where each element of the partition of an agent is to be interpreted as a set of states of the world among which this agent is unable to distinguish at the time of delivery. Radner argues that in this model the possible trades among the agents have to be measurable with respect to the join of their information structures. Our result seems to suggest that this condition is not as convincing as it may seem. If states of the world have to be directly contractible, i.e. verifiable by an institution which enforces contingent contracts, measurability with respect to agents' information structures is neither necessary nor sufficient, the only relevant thing being that all transactions be measurable with respect to the information structure of the institution.

We end this note by mentioning that additional problems arise if we consider implementing a social choice correspondence (SCC). In this case the SCC must satisfy a closedness requirement; however, it can be shown by means of simple examples that the Pareto and the Arrow-Debreu correspondences do not satisfy this requirement and, hence, are not implementable. 


\section{Acknowledgements}

We thank F. Forges, A. Mas-Colell, R. Repullo, A. Sen and W. Trockel for helpful comments. Financial support from the IVIE, the Spanish government (DGICyT projects PB93-940 and PB941504), the EU (through a TMR grant under Contract No. ERBFMBICT960626 at the University of Alicante) and SFB 303 at the University of Bonn, are gratefully acknowledged. Any remaining errors are our own.

\section{References}

Abreu, D., Sen, A., 1991. Virtual implementation in Nash equilibrium. Econometrica 59 (4), 997-1021.

Bernheim, B.D., Whinston, M.D., 1998. Incomplete contracts and strategic ambiguity. American Economic Review 88 (4), 902-932.

Maskin, E., 1977. Nash Equilibrium and Welfare Optimality, Mimeo, MIT.

Maskin, E., 1985. The theory of implementation in Nash equilibrium: a survey. In: Hurwicz, L., Schmeidler, D., Sonnenschein, H. (Eds.), Social Goals and Social Organization: Essays in Honor of Elisha A. Pazner, Cambridge University Press, Cambridge, MA, pp. 173-204, Chapter 6.

Matsushima, H., 1988. A new approach to the implementation problem. Journal of Economic Theory 45 (1), $126-144$.

Moore, J., 1992. Implementation, contracts and renegotiation in environments with complete information. In: Laffont, J.-J. (Ed.), Advances in Economic Theory, Vol. 1, Cambridge University Press, Cambridge, MA.

Palfrey, T.R., Srivastava, S., 1989. Implementation with incomplete information in exchange economies. Econometrica 57 (1), 115-134.

Radner, R., 1968. Competitive equilibrium under uncertainty. Econometrica 36 (1), 31-58. 